\title{
Impactos potenciais das mudanças propostas no Código Florestal Brasileiro sobre os répteis brasileiros
}

\author{
Otavio Augusto Vuolo Marques ${ }^{1,5}$, Cristiano Nogueira ${ }^{2}$, Marcio Martins $^{3}$ \& Ricardo Jannini Sawaya ${ }^{4}$ \\ ${ }^{1}$ Laboratório de Ecologia e Evolução, Instituto Butantan, \\ Av. Dr. Vital Brasil, n. 1500, CEP 05503-900, São Paulo, SP, Brasil \\ ${ }_{2}^{2}$ Departamento de Zoologia, Universidade de Brasília - UnB, CEP 70910-900, Brasília, DF, Brasil \\ ${ }^{3}$ Departamento de Ecologia, Instituto de Biociências, \\ Universidade de São Paulo - USP, CEP 05508-090, São Paulo, SP, Brasil \\ ${ }^{4}$ Departamento de Ciências Biológicas, Universidade Federal de São Paulo - UNIFESP, \\ Rua Prof. Artur Riedel, n. 275, CEP 09972-270, Diadema, SP, Brasil \\ ${ }^{5}$ Autor para correspondência: Otavio Augusto Vuolo Marques, e-mail: otaviomarques@butantan.gov.br
}

MARQUES, O.A.V., NOGUEIRA, C., MARTINS, M. \& SAWAYA, R.J. Potential impacts of changes in the Brazilian Forest Code on reptiles. Biota Neotrop. 10(4): http://www.biotaneotropica.org.br/v10n4/en/ abstract?article+bn00510042010.

\begin{abstract}
We evaluate the potential impacts on Brazilian reptiles (721 species already described), if the proposed changes in the Brazilian Forest Code are approved. The possibility of environmental compensation (a legal obligation in case of disturbance of natural habitats) in basins or micro basins different from that in which the disturbance occurred would have harmful effects on reptile diversity. Some reptile genera include species that rarely co-occur in space. Thus, conservation action planning based on naturally smaller scales, such as micro basins, is most suitable to maintain species composition across large regions. The proposed changes also include the removal of mountaintops as Areas of Permanent Preservation (APP, areas which must be permanently protected, despite the fact that they are not part of a park), as well as a reduction in the width of gallery forests and protected riparian habitats (which are APPs). Many Brazilian reptiles are restricted to high elevation areas, whereas others dwell only or mostly in gallery forests and riparian areas. Thus, the habitat loss that would result from these two changes could make some reptiles vulnerable to extinction. The proposed changes also include allowing the restoration of the Legal Reserves (LR, the reserves of natural vegetation which landowners have to keep in private areas) using exotic plant species. There are evidences that many Brazilian reptiles are not able to persist in human-modified environments like forests composed of exotic trees. The proposed changes also allow the compensation of disturbances imposed on LR inside existing protected areas. However, existing protected areas are not sufficient for the maintenance of reptile diversity in Brazil (mainly because many species have restricted distributions). If approved, the proposed changes in the code will impose significant negative effects on the Brazilian reptile fauna, an important component of the country's natural heritage. Furthermore, unknown molecules with potential for pharmaceutical use could also be lost.

Keywords: reptiles, Brazilian Forest Code, habitat loss, extinction.
\end{abstract}

MARQUES, O.A.V., NOGUEIRA, C., MARTINS, M. \& SAWAYA, R.J. Impactos potenciais das mudanças propostas no Código Florestal Brasileiro sobre os répteis brasileiros. Biota Neotrop. 10(4): http://www. biotaneotropica.org.br/v10n4/pt/abstract?article+bn00510042010.

Resumo: Avaliamos os impactos potenciais sobre a fauna brasileira de répteis (721 espécies descritas até o momento), caso sejam adotadas mudanças propostas por um substitutivo do atual Código Florestal Brasileiro. A possibilidade de compensação ambiental (obrigação legal no caso de degradação de habitats naturais) em bacias ou microbacias distintas daquelas degradadas seria uma das modificações do código vigente que prejudicaria a manutenção da diversidade de répteis. Alguns gêneros de répteis são compostos por espécies que raramente co-ocorrem em uma mesma área. Assim, ações de conservação em escalas reduzidas, em unidades naturais como microbacias, seriam mais adequadas para representar a variação da composição de espécies entre áreas. O substitutivo prevê a exclusão de topos de montanhas como Área de Preservação Permanente (APP), bem como a redução da largura das matas marginais a cursos d’água (que também são APPs). Diversos répteis brasileiros estão restritos a áreas de altitude, ao passo que outros vivem somente ou principalmente em matas de galeria ou áreas ripárias. Assim, a perda de habitat nessas áreas deve tornar alguns répteis vulneráveis a extinção. A proposta também autoriza a recuperação de Reservas Legais (RL) usando espécies de plantas exóticas. Há evidências que muitos répteis brasileiros não conseguem sobreviver em ambientes alterados pelo homem, incluindo as florestas constituídas por espécies exóticas. A proposta também tornará possível compensar RL dentro de unidades de conservação. Entretanto, as unidades de conservação existentes não seriam suficientes para a manutenção da diversidade de répteis no Brasil (principalmente porque muitas espécies têm distribuição restrita). Se adotadas, as mudanças propostas ao Código Florestal Brasileiro terão fortes impactos sobre a fauna de répteis brasileira, um importante componente do patrimônio natural do país. Além disso, moléculas com potencial farmacêutico, presentes nos venenos de muitas espécies, poderão ser perdidas.

Palavras-chave: répteis, Código Florestal Brasileiro, perda de habitat, extinção. 


\section{Introdução}

Das 8.734 espécies de répteis reconhecidas atualmente no mundo (Uetz 2008), 721 ocorrem no Brasil (SBH 2010). Não restam dúvidas de que a diversidade de répteis no Brasil é bem maior, uma vez que mais de 80 espécies foram descritas somente na última década.

Uma análise recente que avalia o estado de conservação de 1.500 espécies de répteis distribuídas pelo mundo indica que cerca de $20 \%$ das espécies é ameaçada de extinção. Esse estudo também ressalta que diversos declínios de espécies ocorreram em áreas tropicais com elevada pressão antrópica e conclui que a perda de hábitat é o principal fator de ameaça de extinção de répteis. No Brasil, apenas 20 espécies são reconhecidas oficialmente como ameaçadas de extinção e a perda e a degradação de habitats são consideradas as principais causas de ameaça (Martins \& Molina 2008). Entretanto, avaliações detalhadas mais recentes, como a realizada para o Estado de São Paulo (Marques et al. 2009), indicam que uma porcentagem maior de répteis estaria ameaçada. Mas ainda está por ser feita uma nova análise cuidadosa do estado de conservação de toda a fauna brasileira de répteis, que provavelmente irá aumentar o número de espécies brasileiras ameaçadas.

Este texto avalia os impactos aos quais a fauna brasileira de répteis estaria sujeita caso fossem postas em prática as mudanças propostas no atual do Código Florestal Brasileiro (CFB), pelo substitutivo do Relator da Comissão Especial da Câmara dos Deputados, de julho de 2010.

\section{Métodos}

Enumeramos, discutimos e ilustramos com exemplos as principais alterações propostas pelo substitutivo do Código Florestal Brasileiro que podem afetar diretamente a fauna de répteis do país. Para subsidiar tais avaliações, utilizamos informações disponíveis em artigos científicos sobre taxonomia, sistemática, distribuição geográfica, uso de habitat e outros aspectos da história natural de várias espécies, bem como a extensa experiência de campo dos autores nos diversos biomas brasileiros.

\section{Resultados e Discussão}

\section{Não obrigatoriedade de compensação ambiental nas mesmas bacias ou microbacias}

Grupos de organismos com padrões de distribuição mais localizados, como os répteis, tendem a ser mal representados em estratégias de conservação de foco amplo, baseadas em grandes unidades da paisagem (ver Araújo et al. 2001). Alguns gêneros de répteis são compostos por espécies que raramente co-ocorrem em mesma área. Assim, ações de conservação em escalas reduzidas, em unidades naturais como microbacias, são mais adequadas para representar a variação da composição de espécies entre áreas (ver Nogueira et al. 2010a, para estudo com ictiofauna). Como exemplo, a fauna de lagartos do Cerrado, com alta substituição de espécies entre ambientes e localidades (Costa et al. 2007, 2010, Nogueira 2006, Nogueira et al. 2009), só estaria bem representada em estratégias de conservação que protejam toda a gama de habitats em escala local (Nogueira et al. 2009). A extinção das microbacias como unidade de planejamento acaba favorecendo a concentração de Reservas Legais em blocos únicos, que poderiam deixar de representar a variação da composição de espécies entre áreas.

\section{Os topos de morros, montes, montanhas e serras deixarão de ser considerados Área de Preservação Permanente (APP)}

A existência de habitats originais nas APPs de áreas elevadas garante a preservação de vários endemismos de répteis no país. Entre 80 espécies de serpentes registradas para a Serra do Mar, um total de $12(15 \%)$ está restrito as áreas de altitudes elevadas (ver Marques et al. 2004, Marques 2009). Diversas outras localidades de altitude elevada no Brasil abrigam espécies endêmicas de lagartos, tais como Heterodactylus lundii Reinhardt \& Luetken, 1862, Placosoma cipoense Cunha, 1966 e Rhachysaurus brachylepis (Dixon, 1974), e a degradação de seus habitats naturais aumentaria a vulnerabilidade dessas espécies (Rodrigues 2005). As espécies de répteis de altitude que ocorrem no território brasileiro também possuem usualmente distribuição geográfica reduzida, o que as torna mais susceptíveis caso seu ambiente natural não seja protegido.

\section{Redução da largura de Área de Preservação Permanente (APP) marginais a cursos d'água}

Um total de 60 espécies de serpentes habitam as matas que margeiam cursos d'água que entrecortam o Cerrado brasileiro, o que representa cerca de $40 \%$ da biodiversidade de serpentes deste bioma (Marques et al. in press). Pelo menos metade dessas espécies depende dessas matas para a sua sobrevivência. A redução de matas ciliares e outros ambientes ripários (como campos úmidos), que já constituem ambientes muito restritos no Cerrado, provavelmente tornará ameaçadas muitas dessas espécies que hoje não se encontram em perigo. Entre elas, vale ressaltar a serpente Anilius scytale (Linnaeus, 1758), que vive associada a florestas, e no Cerrado ocorre exclusivamente em matas ciliares (Marques et al. in press.). $\mathrm{O}$ aumento da vulnerabilidade dessa espécie em função da diminuição de seu hábitat não representa somente a ameaça a uma espécie, mas de uma família inteira de serpentes no Cerrado. Família esta de grande importância do ponto de vista evolutivo, já que representa uma linhagem antiga, possivelmente muito similar ao ancestral de todas as existentes no mundo (ver Wiens et al. 2008).

\section{Recuperar Reservas Legais com espécies vegetais exóticas}

A perda ou modificação de habitats nativos é a maior fonte global de ameaça à biodiversidade (IUCN 2010). Uma grande parte dos répteis brasileiros não é capaz de sobreviver em ambientes muito alterados pelo homem, incluindo as florestas constituídas de espécies exóticas (que são, em geral, muito distintas dos ambientes naturais em diversidade e estrutura). Um estudo realizado na região de Itirapina (Spina \& Martins, não publicado), interior de São Paulo, possibilitou a comparação da herpetofauna de uma área preservada de campo cerrado com a de uma área contígua de plantação de eucalipto. Menos de um terço das espécies encontradas no campo cerrado adjacente foi encontrada dentro do eucaliptal, evidenciando o forte efeito negativo advindo da substituição da vegetação nativa por uma plantação de espécies exóticas. Em uma área de Mata Atlântica no litoral Sul de São Paulo, na região da Juréia, a substituição da mata por bananais acarretou na ocorrência de menor abundância de serpentes arborícolas do gênero Chironius (Marques 1998, Marques \& Sazima 2004). Essas serpentes, assim como outros répteis arborícolas parecem depender fortemente das condições estruturais da mata original, que inclui grande número de bromélias, outras epífitas e lianas (Marques 1998, Marques \& Sazima 2004).

\section{Torna-se possível compensar Reservas Legais dentro de Unidades de Conservação (UC)}

Embora vitais para a biodiversidade, UC não devem ser vistas como única opção para a conservação dos répteis brasileiros, pois várias das espécies conhecidas ocorrem fora da rede atual de áreas protegidas. Assim, são necessárias ações complementares para assegurar a proteção dentro e fora de parques e reservas. Sabe-se que o estabelecimento de UC em regiões produtivas e sob alta pressão de ocupação humana envolve altos custos (Brooks et al. 2004, Margules \& Pressey 2000). Na região do Cerrado, por exemplo, são raras as UC em regiões de solos planos, em topos de chapada, embora grande parte da riqueza de lagartos deste bioma esteja concentrada exatamente nestes ambientes 
(ver Nogueira 2006, Nogueira et al. 2009), altamente visados pela agricultura (Cavalcanti \& Joly 2002, Klink \& Machado 2005). Nestes casos a conservação de ambientes naturais em regiões sob alta pressão de atividades econômicas depende da manutenção de reservas legais em terras privadas, fora de áreas protegidas. Além disso, mesmo atingindo a meta global da Convenção da Diversidade Biológica de proteção de $10 \%$ de cada bioma na forma de UC, estes não serão suficientes para a manutenção da diversidade de répteis no Brasil. Isto porque essas áreas não seriam representativas em termos biogeográficos, pois tenderiam a se concentrar em algumas localidades em detrimento de outras. Considerando a variação de habitats e a variação na distribuição local de espécies, para garantir a conservação de répteis ao longo da grande variedade de paisagens que compõem os biomas brasileiros, a compensação de reservas legais deveria ocorrer na própria microbacia onde as espécies deveriam ser protegidas.

\section{Considerações Finais}

Como mostrado acima, caso fossem postas em prática, as mudanças propostas ao Código Florestal Brasileiro teriam fortes impactos sobre a fauna de répteis brasileira. Correríamos o risco de perder diversas espécies, o que vai diametralmente contra os objetivos centrais da Convenção da Diversidade Biológica e representaria uma perda incalculável para o país.

Entre as espécies que correm o risco de desaparecer estão algumas que poderiam fornecer moléculas com potencial farmacêutico, como as serpentes venenosas. No passado, uma molécula descoberta no veneno da jararaca comum (Bothrops jararaca Wied, 1824), endêmica da Mata Atlântica, deu origem ao medicamento captopril, um anti-hipertensivo que beneficia milhões de vidas humanas e que garantiu faturamento de bilhões de dólares à multinacional Squibb (Gambardella 1995). A maior parte das serpentes brasileiras ainda tem seus venenos pouco estudado e é certo que moléculas ainda desconhecidas serão perdidas com a extinção destas espécies.

\section{Agradecimentos}

Agradecemos ao programa BIOTA-FAPESP pelo convite para elaboração desse manuscrito. Ao CNPq (INCTTOX e auxílios a OAVM, MM e RJ), CAPES (auxílio a CN) e FAPESP (Procs. 08/54472-2, de RJS e 10/50146-3, de MM) pelo apoio financeiro.

\section{Referências Bibliográficas}

ARAÚJO, M.B., HUMPHRIES, C.J., DENSHAM, P.J., LAMPINEN, R., HAGEMEIJER, W.J.M., MITCHELL-JONES, A.J. \& GASC, J.P. 2001. Would environmental diversity be a good surrogate for species diversity? Ecography 24(1):103-110.

BROOKS, T., FONSECA, G.A.B. \& RODRIGUES, A.S.L. 2004. Species, data, and conservation planning. Conserv. Biol. 18(6):1682-1688.

CAVALCANTI, R.B. \& JOLY, C.A. 2002. Biodiversity and Conservation priorities in the Cerrado region. In The Cerrados of Brazil - Ecology and Natural History of a Neotropical Savanna. (P.S. Oliveira \& R.J. Marquis, ed.). Columbia University Press, New York, p.351-367.

COSTA, G.C., NOGUEIRA, C., MACHADO, R.B. \& COLLI, G.R. 2007. Squamate richness in the Brazilian Cerrado and its environmental-climatic associations. Divers. Distrib. 13(6):714-724.

COSTA, G.C., NOGUEIRA, C., MACHADO, R.B. \& COLLI, G.R. 2010 Sampling bias and the use of ecological niche modeling in conservation planning: a field evaluation in a biodiversity hotspot. Biodivers. Conserv. 19(3):883-899.
GAMBARDELLA, A. 1995. Science and innovation: the US pharmaceutical industry during the 1980s. Cambridge University Press, Cambridge.

INTERNATIONAL UNION FOR CONSERVATION OF NATURE - IUCN. 2010. http://www.iucn.org/ (último acesso em 30/08/2010).

KLINK, C.A. \& MACHADO, R.B. 2005. Conservation of the Brazilian Cerrado. Conserv. Biol. 19(3):707-713.

MARGULES, C.R. \& PRESSEY, R.L. 2000. Systematic conservation planning. Nature 405(6783):243-253.

MARQUES, O.A.V. \& SAZIMA, I. 2004. História natural dos répteis da Estação Ecológica Juréia-Itatins. In Estação Ecológica Juréia-Itatins: ambiente físico, flora e fauna (O.A.V. Marques \& W. Duleba, ed.). Holos Editora, Ribeirão Preto, p.257-277.

MARQUES, O.A.V. 1998. Composição faunística, história natural e ecologia de serpentes da Mata Atlântica na Estação Ecológica Juréia-Itatins, SP. Tese de Doutorado, Universidade de São Paulo, São Paulo.

MARQUES, O.A.V. 2009. A fauna de répteis na região de Paranapiacaba. In Patrimônio da Reserva Biológica do Alto da Serra de Paranapiacaba - a antiga Estação Biológica do Alto da Serra (M.I.M.S. Lopes, K. Kirizawa \& M.M.R.F. Melo, org.). São Paulo, Editora Neotropica, p.607-620.

MARQUES, O.A.V., ETEROVIC, A. \& SAZIMA, I. 2004. Snakes of the Brazilian Atlantic Forest: an illustrated field guide for the Serra do Mar range. Holos Editora, Ribeirão Preto, 205p.

MARQUES, O.A.V., ETEROVIC, A., NOGUEIRA, C. \& SAZIMA, I. in press. Serpentes do Cerrado: guia ilustrado. Holos Editora, Ribeirão Preto.

MARQUES, O.A.V., NOGUEIRA, C., SAWAYA, R.J., BÉRNILS, R.S., MARTINS, M., MOLINA, F., FERRAREZZI, H., FRANCO, F.L. \& GERMANO, V.J. 2009. Répteis. In Livro vermelho da fauna ameaçada de extinção do Estado de São Paulo (C. Kierulff, org.). SEMA, São Paulo, p.285-327.

MARTINS, M. \& MOLINA, F.B. 2008. Panorama geral dos répteis ameaçados do Brasil. In Livro vermelho da Fauna Brasileira ameaçada de extinção (A.B.M. Machado, G.M. Drummond, A.P. Paglia, ed.). MMA, Brasília, Fundação Biodiversitas, Belo Horizonte, p.327-334.

NOGUEIRA, C. 2006. Diversidade e padrões de distribuição da fauna de lagartos do Cerrado. Tese de Doutorado, Universidade de São Paulo, São Paulo.

NOGUEIRA, C., BUCKUP, P.A., MENEZES, N.A., OYAKAWA, O.T., KASECKER, T.P., RAMOS NETO, M.B. \& DA SILVA. J.M.C. 2010a. Restricted-Range Fishes and the Conservation of Brazilian Freshwaters. PLoS One 5:e11390.

NOGUEIRA, C., COLLI, G.R., COSTA G.C. \& MACHADO R.B. $2010 \mathrm{~b}$. Diversidade de répteis Squamata e evolução do conhecimento faunístico no Cerrado. In Cerrado: conhecimento científico quantitativo como subsídio para ações de conservação. (I.R. Diniz, J. Marinho-Filho, R.B. Machado \& R.B. Cavalcanti, ed.). Editora UnB, Brasília, p.333-375.

NOGUEIRA, C., COLLI, G.R. \& MARTINS, M. 2009. Local richness and distribution of the lizard fauna in natural habitat mosaics of the Brazilian Cerrado. Austral Ecol. 34:83-96.

RODRIGUES, M.T. 2005. Conservação dos répteis brasileiros: os desafios para um país megadiverso. Megadiversidade 1(1):87-94.

SOCIEDADE BRASILEIRA DE HERPETOLOGIA - SBH. 2010. Brazilian reptiles - List of species. http://www.sbherpetologia.org.br (último acesso em 20/08/2010).

UETZ, P. 2008. The EMBL Reptile Database. http://www.reptile-database. org/db-info/SpeciesStat.html (último acesso em 20/08/2010).

WIENS, J.J., KUCZYNSKI, C.A., SMITH, A., MULCAHY, D.G., SITES Jr., J.W., TOWNSEND, T.M. \& REEDER, T.W. 2008. Branch lengths, support, and congruence: testing the phylogenomic approach with 20 nuclear loci in snakes. Syst. Biol. 57(3):420-431. 
